\title{
SOME CONSIDERATIONS ABOUT REACHABILITY OF SWITCHED LINEAR SINGULAR SYSTEMS
}

\author{
Josep Clotet and M. Dolors Magret \\ Departament de Matemàtica Aplicada I \\ ETSEIB, UPC, Spain \\ e-mail: josep.clotet@upc.edu \\ m.dolors.magret@upc.edu
}

\begin{abstract}
No necessary and sufficient condition for reachability of switched linear singular systems has been found, exceptuating the case of the so-called "equisingular systems". Such a condition is not valid in the general case, as examples show.
\end{abstract}

\section{Introduction}

The study of characterization of reachability in the case of switched linear systems, including those which are singular, arises from the practical importance of such systems. In the case of non-singular switched linear systems, an algebraic characterization can be found in [6]. Necessary and sufficient conditions (but not necessary and sufficient) are provided in [4] and [5]. A necessary and sufficient condition for the so-called "equisingular" systems is provided in [1]. This condition cannot be generalized to systems which are not "equisingular", as the example in the last section shows.

Received: May 11, 2015; Accepted: August 8, 2015

2010 Mathematics Subject Classification: 93B05, 93B25, 93B27.

Keywords and phrases: switched linear system, reachability.

Communicated by K. K. Azad 
The structure of the paper is as follows:

In Section 2, we summarize the definitions, we need of reachability and switched linear singular systems.

In Section 3, we recall the different results obtained by different authors, including the algebraic characterization by the authors of reachability for “equisingular” linear systems.

In Section 4, we consider an example which shows the impossibility to generalize the algebraic characterization obtained for "equisingular" systems.

Throughout the paper, $\mathbb{R}$ will denote the set of real numbers, $M_{n \times m}(\mathbb{R})$ the set of matrices having $n$ rows and $m$ columns and entries in $\mathbb{R}$ (in the case where $n=m$, we will simply write $\left.M_{n}(\mathbb{R})\right)$ and by $G_{n}(\mathbb{R})$ the group of non-singular matrices in $M_{n}(\mathbb{R})$.

\section{Preliminaries}

Switched linear systems consist of different subsystems of linear equations and a rule providing the switching between them. In the case where at least one of the subsystems is singular, it is called switched linear singular system.

Definition 1. A switched linear singular system $\sum$ is a system which consists of several linear subsystems and a rule that determines the switching between them.

It can be written as

$$
\left\{\begin{array}{l}
E_{\sigma(t)^{x}}(t)=A_{\sigma(t)^{X}} x(t)+B_{\sigma(t)^{u}}{ }_{\sigma(t)}(t), \\
y(t)=C_{\sigma(t)^{x}}(t)
\end{array}\right.
$$

where $x(t) \in \mathbb{R}^{n}$ is the state, $y(t) \in \mathbb{R}^{q}$ is the output, $\sigma:\left[t_{0}, T\right) \rightarrow M$, where $t_{0}$ is the initial time, $t_{0}<T \leq \infty, M=\{1, \ldots, m\}$ is a right-continuous 
piecewise constant mapping (well-defined switching path), $u_{i}(t) \in \mathbb{R}^{p}$ is the input function, $E_{i}, A_{i} \in M_{n}(\mathbb{R}), \quad B_{i} \in M_{n \times p}(\mathbb{R}), \quad C_{i} \in M_{q \times n}(\mathbb{R}), \quad i \in M$ and at least one of the matrices $E_{i}$ is a singular matrix $\left(r k\left(E_{i}\right)<n\right)$.

Let $x\left(t ; t_{0}, x_{0}, u, \sigma\right)$ be the solution $x(t)$ in the time $t$ of system (1), with an initial condition $x\left(t_{0}\right)=x_{0}$, input $u$ and well-defined switching path $\sigma$.

We will assume from now on that for all $i \in M$, there exists $\lambda_{i} \in \mathbb{C}$ such that $\operatorname{det}\left(\lambda_{i} E_{i}-A_{i}\right) \neq 0$ (the subsystems are regular).

For all linear subsystems, we can consider a standard decomposition. That is to say, there exist $P_{i}, Q_{i} \in G l_{n}(\mathbb{R})$ such that:

$$
P_{i} E_{i} Q_{i}=\left(\begin{array}{cc}
I_{n_{i}} & 0 \\
0 & \mathcal{N}_{i}
\end{array}\right), \quad P_{i} A_{i} Q_{i}=\left(\begin{array}{cc}
G_{i} & 0 \\
0 & I_{n-n_{i}}
\end{array}\right), \quad P_{i} B_{i}=\left(\begin{array}{c}
B_{i, 1} \\
B_{i, 2}
\end{array}\right),
$$

where $\mathcal{N}_{i} \in M_{\left(n-n_{i}\right) \times\left(n-n_{i}\right)}(\mathbb{R})$ is a nilpotent matrix with nilpotent index $h_{i}, G_{i} \in M_{n_{i} \times n_{i}}(\mathbb{R})$. Note that $r k\left(E_{i}\right)=n, P_{i}=E_{i}^{-1}, Q_{i}=I_{n}, n_{i}=n$ and $G_{i}=E_{i}^{-1} A_{i}$. Let us denote by $h$ the maximum of the nilpotent indices of matrices $\mathcal{N}_{i}$, for all singular subsystems.

Definition 2. System $\sum$ :

$$
\left\{\begin{array}{l}
E_{\sigma(t)^{\dot{x}}}(t)=A_{\sigma(t)^{x}}(t)+B_{\sigma(t)^{u}}{ }_{\sigma(t)}(t) \\
y(t)=C_{\sigma(t)^{x}}(t)
\end{array}\right.
$$

is said to be (completely) reachable if for any given initial time $t_{0} \in \mathbb{R}$ and state $x_{f} \in \mathbb{R}^{n}$, there exists a real number $t_{f}>t_{0}$, a well-defined switching path $\sigma:\left[t_{0}, t_{f}\right] \rightarrow M$ and an input $u:\left[t_{0}, t_{f}\right] \rightarrow \mathbb{R}^{p}$ such that $x_{f}=$ $x\left(t_{f} ; t_{0}, 0, u, \sigma\right)$. 
System $\sum$ :

$$
\left\{\begin{array}{l}
E_{\sigma(t)^{x}} \dot{x}(t)=A_{\sigma(t)^{X}} x(t)+B_{\sigma(t)^{u}}{ }_{\sigma(t)}(t), \\
y(t)=C_{\sigma(t)^{x}}(t)
\end{array}\right.
$$

is said to be (completely) controllable if for any given initial time $t_{0} \in \mathbb{R}$ and initial state $x_{0} \in \mathbb{R}^{n}$, there exists a real number $t_{f}>t_{0}$, a well-defined switching path $\sigma:\left[t_{0}, t_{f}\right] \rightarrow M$ and an input $u:\left[t_{0}, t_{f}\right] \rightarrow \mathbb{R}^{p}$ such that $x_{f}=x\left(t_{f} ; t_{0}, 0, u, \sigma\right)$.

\section{Results on Reachability and Controllability}

We first recall the definition of admissible controls. Let us denote by $t_{11}, t_{12}, \ldots, t_{1 k}$ the $k$ switching discontinuous points in any given time interval $\left[t_{1}, t_{2}\right], t_{1}<t_{11}<t_{12}<\cdots<t_{1 k}<t_{2}$. That is to say, $\sigma(t)=\sigma\left(t_{1}\right)$ for $t \in\left[t_{1}, t_{11}\right), \quad \sigma(t)=\sigma\left(t_{11}\right)$ for $t \in\left[t_{11}, t_{12}\right), \ldots, \sigma(t)=\sigma\left(t_{1 k}\right)$ for $t \in$ $\left[t_{1 k}, t_{2}\right]$. Then the set of admissible controls in $\left[t_{1}, t_{2}\right]$ is the set:

$$
\mathcal{U}_{\sigma}\left[t_{1}, t_{2}\right]=\left\{u=\left(u_{1}|\ldots| u_{m}\right)\right\}
$$

with $u_{\sigma\left(t_{1 j}\right)}$ an $(h-1)$-continuously differentiable function in the interval $\left[t_{1 j}, t_{1(j+1)}\right], 0 \leq j \leq k, t_{10}=t_{1}, t_{1(k+1)}=t_{2}$ such that

$$
\begin{aligned}
& \sum_{r=0}^{h_{\sigma\left(t_{1 j}\right)^{-1}}} \mathcal{N}_{\sigma\left(t_{1 j}\right)}^{r} B_{\sigma\left(t_{1 j}\right), 2} u_{\sigma\left(t_{1 j}\right)}^{(r)}\left(t_{1 j}^{+}\right) \\
& =-\left(\left(0 \mid I_{n-n_{\sigma\left(t_{1 j}\right)}}\right) Q_{\sigma\left(t_{1 j}\right)}^{-1}\right) x\left(t_{1 j}^{-} ; t_{1}, x_{0}, u, \sigma\right),
\end{aligned}
$$

$0 \leq j \leq k, t_{10}^{-}=t_{1}, u_{\sigma\left(t_{1 j}\right)}^{(r)}\left(t_{1 j}^{+}\right)$the $r$-derivative of $u_{\sigma\left(t_{1 j}\right)}(t)$ at $t=t_{1 j}$ and $x\left(t_{1 j}^{-} ; t_{1}, x_{0}, u, \sigma\right)$ the left limit of $x\left(t ; t_{1}, x_{0}, u, \sigma\right)$ at $t=t_{1 j}$. 
Note that this set of admissible controls does not necessarily exist.

We can summarize some of the results which are known with respect to conditions which ensure reachability or controllability of switched linear singular systems as follows:

(i) Meng-Zhang [4]: a necessary condition for switched linear singular systems to be (completely) reachable, accepting only admissible controls.

(ii) Meng-Zhang [5]: a sufficient condition for switched linear singular systems to be (completely) reachable, accepting only admissible controls.

(iii) Meng-Zhang [5]: a necessary condition for switched linear singular systems to be (completely) controllable, accepting only admissible controls.

(iv) Meng-Zhang [5]: a sufficient condition for switched linear singular systems to be (completely) controllable, accepting only admissible controls.

(v) Clotet et al. [1]: a necessary and sufficient condition for switched linear singular systems assuming $r k\left(E_{i}\right)<n$ for all $i \in M$, which satisfy the "equisingularity condition" to be (completely) reachable/(completely) controllable, with the controls not necessarily admissible.

(vi) Clotet-Magret [2]: a sufficient condition for switched linear singular systems with two subsystems satisfying certain conditions, to be (completely) reachable, not assuming the controls to be admissible.

(vii) Clotet et al. [3]: a necessary condition for switched linear singular systems where $r k\left(E_{i}\right)<n$ for all $i \in M$, to be (completely) reachable, not requiring the controls to be admissible.

More concretely, we will state the results obtained by these different authors. In order to do that, we first need to introduce some further notations.

In the following, given two matrices $M \in M_{p}(\mathbb{R}), \quad N \in M_{p \times q}(\mathbb{R})$, we will denote by $\langle M \mid N\rangle$ the vector subspace $\operatorname{Im}\left[N, M N, M^{2} N, \ldots, M^{p-1} N\right]$.

Let us consider the following vector subspaces (see [4]): 


$$
\mathcal{V}_{1}=\sum_{i=1}^{m} Q_{i}\left(\left\langle G_{i} \mid B_{i, 1}\right\rangle \oplus\left\langle\mathcal{N}_{i} \mid B_{i, 2}\right\rangle\right)
$$

and for $k>1$,

$$
\mathcal{V}_{k}=\sum_{i=1}^{m} Q_{i}\left(\left\langle G_{i} \mid\left(\left(I_{n_{i}} \mid 0\right) Q_{i}^{-1}\right) \mathcal{V}_{k-1}\right\rangle \oplus\left\langle\mathcal{N}_{i} \mid B_{i, 2}\right\rangle\right) .
$$

We have $\mathcal{V}_{1} \subseteq \mathcal{V}_{2} \subseteq \cdots \subseteq \mathcal{V}_{n}$. If there exists $1<i \leq n$ such that $\mathcal{V}_{i}=\mathcal{V}_{i-1}$, then for all $\ell>i, \mathcal{V}_{\ell}=\mathcal{V}_{i}$.

Theorem 1 [4]. If a switched linear singular system $\sum$ is (completely) reachable, then $\mathcal{V}_{n}=\mathbb{R}^{n}$.

Theorem 2 [5]. If $\mathcal{V}_{n}=\mathbb{R}^{n}$ and $\left\langle\mathcal{N}_{i} \mid B_{i, 2}\right\rangle=\mathbb{R}^{n-n_{i}}$ for all $i \in M$, then the switched linear singular system $\sum$ is (completely) reachable.

Theorem 3 [5]. If a switched linear singular system $\sum$ is (completely) controllable, then $\mathcal{V}_{n}=\mathbb{R}^{n}$.

Theorem 4 [5]. If $\mathcal{V}_{n}=\mathbb{R}^{n}$ and $\left\langle\mathcal{N}_{i} \mid B_{i, 2}\right\rangle=\mathbb{R}^{n-n_{i}}$ for all $i \in M$, then the switched linear singular system $\sum$ is (completely) controllable.

Theorem 5 [1]. Let us assume that "equisingularity condition" holds $\left(n_{1}=n_{2}=\cdots=n_{m}<n\right)$. Then the switched linear singular system $\Sigma$ is (completely) reachable/(completely) controllable if and only if

$$
\bigcup_{i \in\{1, \ldots, m\}}\left(\left(I_{n_{i}} \mid 0\right) \mathcal{V}_{n} \oplus\left\langle\mathcal{N}_{i} \mid B_{i, 2}\right\rangle\right)=\mathbb{R}^{n}
$$

Theorem 6 [2]. Let us assume $m=2$. That is to say, $M=\{1,2\}$. Let us assume that $\mathcal{V}_{1}=\mathbb{R}^{n}$ and there exists $i_{0} \in M$ such that $\left\langle\mathcal{N}_{i_{0}} \mid B_{i_{0}, 2}\right\rangle=$ $\mathbb{R}^{n-n_{i_{0}}}$. Then the switched linear singular system $\sum$ is (completely) reachable. 
Theorem 7 [3]. Let us assume that $r k\left(E_{i}\right)<n$ for all $i \in M$. If the system $\sum$ is (completely) reachable, then $\mathcal{V}_{n}=\mathbb{R}^{n}$ and there exists $i_{0} \in M$ such that $\left\langle\mathcal{N}_{i_{0}} \mid B_{i_{0}, 2}\right\rangle=\mathbb{R}^{n-n_{i_{0}}}$.

Remark. Though the result above was stated for $m=2$ in [3], it is obvious that it is also true for $m>2$.

Remark. The condition in Theorem 7 is not a sufficient condition, even in the case where only admissible controls were considered.

\section{Illustrative Example}

Let us consider a switched linear singular system consisting of two subsystems, both of them being singular, with $n=8$ :

$$
\begin{aligned}
& G_{1}=\left(\begin{array}{cccc}
0 & 0 & 0 & 0 \\
1 & -1 & 0 & 0 \\
0 & 1 & 0 & 0 \\
0 & 0 & 1 & 0
\end{array}\right), \quad B_{1,1}=\left(\begin{array}{l}
0 \\
0 \\
0 \\
1
\end{array}\right), \quad \mathcal{N}_{1}=\left(\begin{array}{llll}
0 & 0 & 0 & 0 \\
1 & 0 & 0 & 0 \\
0 & 1 & 0 & 0 \\
0 & 0 & 1 & 0
\end{array}\right), \quad B_{1,2}=\left(\begin{array}{l}
1 \\
0 \\
0 \\
0
\end{array}\right) \\
& G_{2}=(1), \quad B_{2,1}=(1), \quad \mathcal{N}_{2}=\left(\begin{array}{ccccccc}
0 & 0 & 0 & 0 & 0 & 0 & 0 \\
0 & 0 & 0 & 0 & 0 & 0 & 0 \\
0 & 0 & 0 & 0 & 0 & 0 & 0 \\
0 & 0 & 0 & 0 & 0 & 0 & 0 \\
0 & 0 & 0 & 0 & 0 & 0 & 0 \\
1 & 0 & 0 & 0 & 0 & 0 & 0 \\
0 & 1 & 0 & 0 & 0 & 0 & 0
\end{array}\right), \quad B_{2,2}=\left(\begin{array}{l}
0 \\
0 \\
1 \\
0 \\
0 \\
0 \\
0
\end{array}\right) \text {. }
\end{aligned}
$$

Straightforward computations show that

$$
\left\langle G_{1} \mid B_{1,1}\right\rangle=\operatorname{Im}\left[\begin{array}{llll}
0 & 0 & 0 & 0 \\
0 & 0 & 0 & 0 \\
0 & 0 & 0 & 0 \\
1 & 0 & 0 & 0
\end{array}\right], \quad\left\langle\mathcal{N}_{1} \mid B_{1,2}\right\rangle=\operatorname{Im}\left[\begin{array}{cccc}
1 & 0 & 0 & 0 \\
0 & 1 & 0 & 0 \\
0 & 0 & 1 & 0 \\
0 & 0 & 0 & 1
\end{array}\right]
$$


so that

$$
\begin{array}{r}
\left\langle G_{1} \mid B_{1,1}\right\rangle \oplus\left\langle\mathcal{N}_{1} \mid B_{1,2}\right\rangle=\operatorname{Im}\left[e_{4}, e_{5}, e_{6}, e_{7}, e_{8}\right], \\
\left\langle G_{2} \mid B_{2,1}\right\rangle=\operatorname{Im}[1], \quad\left\langle\mathcal{N}_{2} \mid B_{2,2}\right\rangle=\operatorname{Im}\left[\begin{array}{l}
0 \\
0 \\
1 \\
0 \\
0 \\
0 \\
0
\end{array}\right]
\end{array}
$$

so that

$$
\left\langle G_{2} \mid B_{2,1}\right\rangle \oplus\left\langle\mathcal{N}_{2} \mid B_{2,2}\right\rangle=\operatorname{Im}\left[e_{1}, e_{4}\right],
$$

where $e_{1}, \ldots, e_{8}$ denote the vectors of the natural basis of $\mathbb{R}^{8}$.

Then

$$
\begin{aligned}
\mathcal{V}_{1} & =\left(\left\langle G_{1} \mid B_{1,1}\right\rangle \oplus\left\langle\mathcal{N}_{1} \mid B_{1,2}\right\rangle\right)+\left(\left\langle G_{2} \mid B_{2,1}\right\rangle \oplus\left\langle\mathcal{N}_{2} \mid B_{2,2}\right\rangle\right) \\
& =\operatorname{Im}\left[e_{1}, e_{4}, e_{5}, e_{6}, e_{7}, e_{8}\right], \\
\mathcal{V}_{2} & =\left(\left\langle G_{1} \mid\left(I_{4} \quad 0\right) \mathcal{V}_{1}\right\rangle \oplus\left\langle\mathcal{N}_{1} \mid B_{1,2}\right\rangle\right)+\left(\left\langle G_{2} \mid\left(I_{1} \quad 0\right) \mathcal{V}_{1}\right\rangle \oplus\left\langle\mathcal{N}_{2} \mid B_{2,2}\right\rangle\right) \\
& =\mathbb{R}^{8} .
\end{aligned}
$$

Therefore $\mathcal{V}_{8}=\mathbb{R}^{8}$.

In this example, the conditions in the statement in Theorem 7 hold: $\left\langle\mathcal{N}_{1} \mid B_{1,2}\right\rangle=\mathbb{R}^{4}$ and $\mathcal{V}_{8}=\mathbb{R}^{8}$. But this system is not (completely) reachable, as it follows from the computation of the reachable states obtained. To obtain them, we need to compute:

$$
e^{G_{1} t}=\left(\begin{array}{cccc}
1 & 0 & 0 & 0 \\
-e^{-t}+1 & e^{-t} & 0 & 0 \\
e^{-t}-1+t & -e^{-t}+1 & 1 & 0 \\
\frac{1}{2} t^{2}-e^{-t}-t+1 & e^{-t}-1+t & t & 1
\end{array}\right), \quad e^{G_{2} t}=\left(e^{t}\right)
$$


There are only three cases to be considered:

Case 1. When subsystem 1 is the only which acts (that is to say, $\sigma:\left[t_{0}, t_{f}\right] \rightarrow M, \sigma\left(t_{0}\right)=1$ for all $\left.t \in\left[t_{0}, t_{f}\right]\right)$, the set of reachable states is

$\mathcal{R}=\left(e^{G_{1}\left(t_{f}-t_{0}\right)}\left(\operatorname{Im}\left[\begin{array}{l}0 \\ 0 \\ 0 \\ 0\end{array}\right]\right)+\left\langle G_{1} \mid B_{1,1}\right\rangle\right) \oplus\left\langle\mathcal{N}_{1} \mid B_{1,2}\right\rangle=\operatorname{Im}\left[e_{4}, e_{5}, e_{6}, e_{7}, e_{8}\right]$

Analogously, in the case where subsystem 1 is the only system which acts, any number of times.

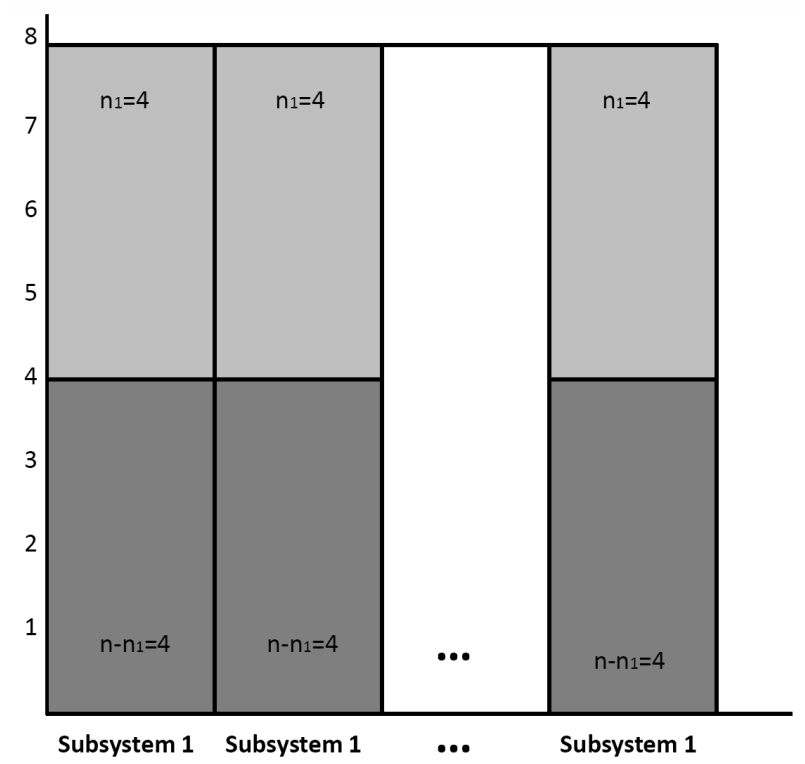

Case 2. In the case where the last subsystem which acts is subsystem 2, that is to say, $\sigma:\left[t_{0}, t_{f}\right] \rightarrow M, \sigma\left(t_{1 k}\right)=2$, for $t \in\left[t_{1 k}, t_{f}\right]$. Then the set of reachable states is:

$$
\mathcal{R}=\left(e^{G_{2}\left(t_{f}-t_{1 k}\right)}(\cdots)+\left\langle G_{2} \mid B_{2,1}\right\rangle\right) \oplus\left\langle\mathcal{N}_{2} \mid B_{2,2}\right\rangle=\operatorname{Im}\left[e_{1}, e_{4}\right] .
$$




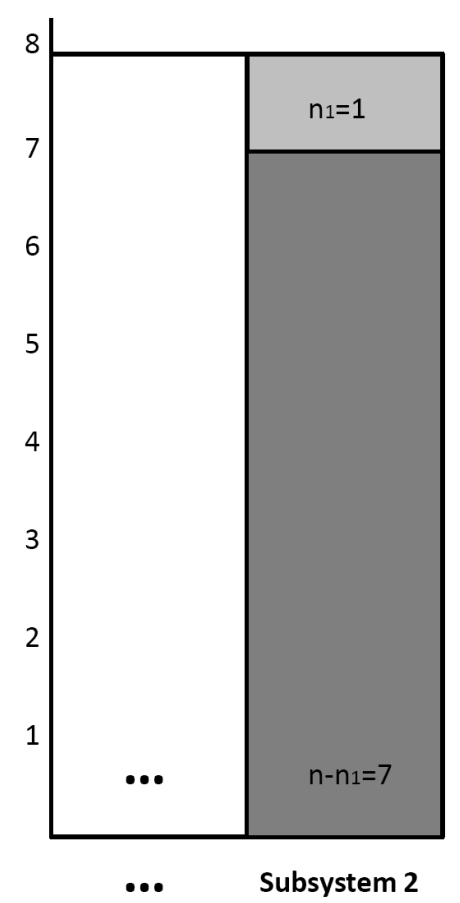

Case 3. If the last subsystem which acts is subsystem 2 and after that subsystem 1 (that is to say, $\sigma:\left[t_{0}, t_{f}\right] \rightarrow M, \sigma\left(t_{1(k-1)}\right)=2$ for $t \in$ $\left[t_{1(k-1)}, t_{1 k}\right), \sigma\left(t_{1 k}\right)=1$ for $\left.t \in\left[t_{1 k}, t_{f}\right]\right)$. Then the set of reachable states is

$$
\begin{aligned}
\mathcal{R} & =\left(e^{G_{1}\left(t_{f}-t_{1 k}\right)}\left(\operatorname{Im}\left[\begin{array}{l}
1 \\
0 \\
0 \\
0
\end{array}\right]+\operatorname{Im}\left[\begin{array}{l}
0 \\
0 \\
0 \\
1
\end{array}\right]\right)+\left\langle G_{1} \mid B_{1,1}\right\rangle\right) \oplus\left\langle\mathcal{N}_{1} \mid B_{1,2}\right\rangle \\
& =\left(e^{G_{1}\left(t_{f}-t_{1 k}\right)}\left(\operatorname{Im}\left[\begin{array}{l}
1 \\
0 \\
0 \\
0
\end{array}\right]\right)+e^{G_{1}\left(t_{f}-t_{1 k}\right)}\left(\operatorname{Im}\left[\begin{array}{l}
0 \\
0 \\
0 \\
1
\end{array}\right]\right)+\left\langle G_{1} \mid B_{1,1}\right\rangle\right) \oplus\left\langle\mathcal{N}_{1} \mid B_{1,2}\right\rangle \\
& =\operatorname{Im}\left[u, e_{4}, e_{5}, e_{6}, e_{7}, e_{8}\right],
\end{aligned}
$$

where $u=\left(1,-e^{-\left(t_{f}-t_{1 k}\right)}+1, e^{-\left(t_{f}-t_{1 k}\right)}-1+\left(t_{f}-t_{1 k}\right), \ldots, 0,0,0,0\right)^{t}$. 
Analogously, in the case where the last subsystem which acts is subsystem 2 and after that subsystem 1 several times.

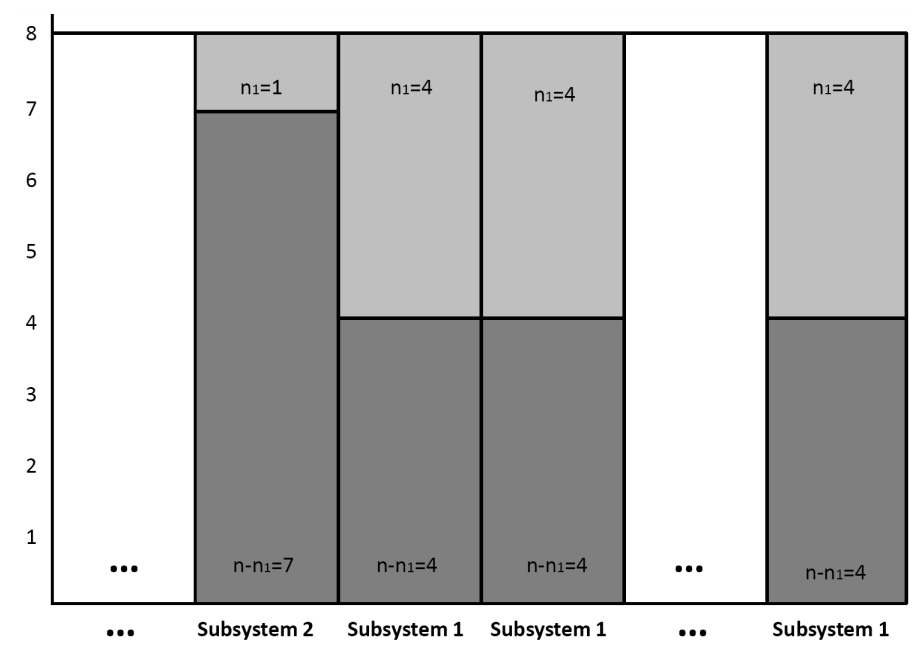

To summarize, the set of reachable states for any well-defined switching path $\sigma:\left[t_{0}, t_{f}\right] \rightarrow M$ will coincide with one of the three cases above. Then there are some states which cannot be reached (for example, $x_{f}=e_{2}$ ). Therefore, the system is not (completely) reachable. Note that this conclusion is true independently whether the controls are required to be admissible or not.

\section{Conclusions}

The necessary condition in Theorem 7 for reachability is not in general a sufficient condition, even in the case of admissible controls. The necessary and sufficient condition in Theorem 5 is not a necessary and sufficient condition if "equisingularity" does not hold, even also in the case of admissible controls. Though, it would be most interesting to obtain such a necessary and sufficient condition, the results obtained up to now and the examples show how little feasible such a condition would be found, independently on whether controls are assumed to be admissible or not. 


\section{References}

[1] J. Clotet, J. Ferrer and M. D. Magret, Switched singular linear systems, Proceedings of the 17th Mediterranean Conference on Control and Automation, Thessaloniki, Greece, 2009, pp. 1343-1347.

[2] J. Clotet and M. D. Magret, Switched singular linear systems and reachability, Book of Abstracts, PHYSCON 2011, León, Spain, 2011, p. 82.

[3] J. Clotet, M. D. Magret and M. E. Montoro, Sistemas lineales conmutados singulares. Accesibilidad, Actas del Congreso, XXIII CEDYA/XIII CMA, Castellón, Spain, 2013, pp. 1047-1054.

[4] B. Meng and J. F. Zhang, Reachability conditions for switched linear singular systems, IEEE Trans. Automat. Control 51 (2006), 482-488.

[5] B. Meng and J. F. Zhang, Controllability conditions for switched linear singular systems, Dyn. Contin. Discrete Impuls. Syst. Ser. B Appl. Algorithms 14 (2007), 641-657.

[6] Z. Sun and S. S. Ge, Switched Linear Systems, Springer-Verlag, London, 2005. 